\title{
Harinas de hojas de plantas aromáticas como fitoterapéuticos en pollos de engorda
}

\author{
Pedro Enrique Lara y Lara(1), Mateo Fabian Itzá Ortiz(1), Edgar Aguilar Urquiso(1) \\ y José Roberto Sanginés García ${ }^{(1)}$
}

${ }^{(1) I n s t i t u t o ~ T e c n o l o ́ g i c o ~ d e ~ C o n k a l, ~ K m ~ 16,3 ~ C a r r e t e r a ~ M e ́ r i d a ~-~ M o t u l, ~ C o n k a l, ~ Y u c a t a ́ n, ~ M e ́ x i c o . ~ E-m a i l: ~ p e d r o . l a r a @ i t c o n k a . e d u . m x, ~}$
mitzaor@prodigy.net.mx, aguilaru_e@yahoo.com.mx, roberto.sangines@itconkal.edu.mx

Resumen - El objetivo del presente trabajo fue evaluar el comportamiento productivo y el rendimiento de la canal en pollos de engorda, con el uso de harina de hojas de plantas aromáticas como aditivo fitoterapéutico al $0,07 \%$ en la dieta. Fueron utilizados 280 pollitos machos de la estirpe Ross 308, de 1 a 42 días de edad, distribuidos de forma aleatoria en un diseño completamente al azar, en cuatro tratamientos y siete repeticiones con 10 pollos cada una. Las combinaciones fueron en proporción 50:50 de harina de Origanum vulgare y Piper auritum (OHS), O. vulgare y Ocimum basilicum (OA), O. basilicum y P. auritum (HSA), y un testigo con flavomicina al 4\%. El grupo testigo obtuvo el mayor peso corporal (2.385 g), consumo de alimento (204 g por ave por día) y mortalidad acumulada $(21,87 \%)$, al final de la prueba. No hubo diferencias con la combinación de OA (2.198 g) y HSA (2.023 g) en peso corporal y consumo de alimento. La combinación OA registró la menor $(1,96)$ y OHS la mayor $(2,44)$ conversión de alimento. No se encontraron diferencias en el rendimiento de la canal. La combinación al 50\% de $O$. vulgare y $O$. basilicum, incluida al $0,07 \%$ en la dieta para pollos de engorda, es una alternativa como promotora del crecimiento y no altera el rendimiento de la canal.

Términos para indexación: Ocimum basilicum, Origanum vulgare, Piper auritum, consumo de alimento, promotores de crecimiento, rendimiento de canal.

\section{Leaf meals of aromatic plants as fitotherapeutics for broilers}

\begin{abstract}
The objective of this study was to determine the growth performance and carcass yield in broilers using leaf meals as an additive phytotherapy of herbs at $0.07 \%$ in the diet. Two hundred eighty male chicks of Ross 308 strain, with 1 to 42 days of age, were randomly assigned in a completely randomized design with four treatments and seven replicates of 10 chickens each. The blends were in the proportion of 50:50 of Origanum vulgare and Piper auritum (OHL), O. vulgare and Ocimum basilicum (OA), O. basilicum and P. auritum (AHL), and one control group with flavomicim at 4\%. The control group showed the highest body weight (2,385 g), feed consumption (204 g per broiler per day) and accumulative mortality (21.87\%), at the end of the assay. There was no difference with OA (2,198 g) and AHL (2,023 g) blends in body weight and feed consumption. The blend OA had the lowest (1.96) and OHL had the highest (2.44) feed conversion. There was no difference in the carcass yield. The blend at $50 \%$ of $O$. vulgare and $O$. basilicum, included at $0.07 \%$ in the diet for broilers, is an alternative for promoting growth and does not alter the yield carcass.
\end{abstract}

Index terms: Ocimum basilicum, Origanum vulgare, Piper auritum, feed consumption, growth promoter, carcass yield.

\section{Introducción}

El uso de antibióticos como promotores de crecimiento (APC) en la producción de alimentos para animales se ha cuestionado durante los últimos años. Los APC, a niveles subterapéuticos, favorecen la selección de factores de resistencia, y los animales que reciben dichas dosis actúan como reservorios de patógenos resistentes los cuales se han detectado en carne o sus subproductos (Kiser, 2007). La creciente demanda de alimentos cárnicos inocuos ha originado la búsqueda denuevas alterativas deAPC, paraincrementar la eficiencia alimenticia animal (Castro, 2005). Los extractos vegetales y aceites esenciales, contenidos en plantas aromáticas, tienen potencial como APC por sus propiedades bactericidas, bacteriostáticas, fungicidas y virales. La actividad antimicrobiana de esos extractos

Pesq. agropec. bras., Brasília, v.45, n.3, p.294-298, mar. 2010 
y aceites ha sido demostrada in vitro (Hernández et al., 2004; Castro, 2005). El orégano (Origanum vulgar) está constituido principalmente por carvacrol y timol (Plaus et al., 2001); hierba santa (Piper auritum, sinónimo de Piper sanctum) contiene fenilpropanoides, lignanos, sequeterpenos y monoterpenos como borneol, alcanfor, cíñelo, eugenol, safrol y una amplia variedad de componentes bencénicos (García Ríos et al., 2007); la albahaca (Ocimum basilicum) contiene aceite esencial (menos de 1\%) de composición compleja, y los componentes del aroma más importantes son el 1,8-cineol, linalol, citral, metilchavicol (estragol), eugenol y metilcinamato (Plaus et al., 2001).

Hernández et al. (2004) encontraron mejora en el consumo de alimento y digestibilidad de la materia seca, en dietas para pollos de engorda suplementadas con aceites esenciales de orégano, canela y pimiento. El uso de harinas tiene la ventaja de ser de fácil elaboración y bajo costo de producción (Ayala et al., 2006).

El objetivo del presente trabajo fue evaluar el comportamiento productivo y el rendimiento de la canal en pollos de engorda, a partir de la combinación de plantas aromáticas, en forma de harina de hoja, incluida al $0,07 \%$ en la dieta como aditivos fitoterapéuticos.

\section{Materiales y Métodos}

El presente estudio se realizó durante los meses de abril y julio en la caseta experimental del Instituto Tecnológico de Conkal (ITC), ubicado en el municipio de Conkal, Yucatán, México, a $20^{\circ} 29^{\prime} \mathrm{N}$ y $89^{\circ} 39^{\prime} \mathrm{W}$, a $8 \mathrm{~m}$ de altitud, con clima tipo Aw, cálido subhúmedo. La precipitación pluvial es de $850 \mathrm{~mm}$ y la temperatura media anual es de $25,5^{\circ} \mathrm{C}$, de acuerdo a la clasificación de Köppen modificada por García (1973).

La caseta avícola experimental contó con piso de cemento y fue dividida en 28 lotes de $1 \mathrm{x} 1 \mathrm{~m}$; cada lote fue equipado con comedero de tolva de $45 \mathrm{~cm}$ de diámetro y bebedero iniciador de $25 \mathrm{~cm}$ de diámetro.

Se utilizaron 280 pollitos machos, de la estirpe Ross 308, desde el día 1 hasta los 42 días de edad, provenientes de un mismo lote de reproductora $\mathrm{y}$ de la misma máquina incubadora; las aves fueron distribuidas de forma aleatoria en cuatro tratamientos, con siete repeticiones de diez pollos cada uno, con densidad de 10 aves $\mathrm{m}^{-2}$ de población inicial. Cada lote experimental recibió calor $\left(32^{\circ} \mathrm{C}\right)$ artificial por una semana, por una criadora de gas modelo Dyc-50 (Santa Catarina, México, DF).

Se evaluaron tres harinas de hojas de plantas: orégano $(O$. vulgare), albahaca (O. basilicum) y hierba santa ( $P$. aurintum), y un testigo con flavomicina al $4 \%$. Las hojas fueron colectadas por las mañanas y secadas en estufa de aire forzado a $60^{\circ} \mathrm{C}$, durante 48 horas; posteriormente, se molieron en un molino de martillos con criba de $4 \mathrm{mmol} \mathrm{L}^{-1}$ (ThomasWiley, EU). Las harinas obtenidas se combinaron en la siguiente proporción porcentual (50:50): $35 \mathrm{~g}$ orégano y $35 \mathrm{~g}$ hierba santa (OHS); $35 \mathrm{~g}$ orégano y $35 \mathrm{~g}$ albahaca (OA); 35 g hierba santa y $35 \mathrm{~g}$ albahaca (HSA). Las combinaciones fueron adicionadas en la dieta al 0,07\%. Los alimentos experimentales se elaboraron con base en sorgo y pasta de soya, en forma de harina, en las dos fases - iniciación 1 a 21 días, y finalización 22 a 42 días (Cuadro 1). Las dietas fueron isoenergéticas e isonitrogenadas, de acuerdo con las recomendaciones sobre los requerimientos nutrimentales para pollos de engorda (National Research Council, 1994).

El alimento y agua fueron ofrecidos ad libitum. El programa de iluminación consistió en un fotoperiodo de luz natural. El programa sanitario y de manejo fue similar para todas las aves recibidas de la planta incubadora sin vacunar, las cuales se vacunó vía oral, en la granja a los ocho días, contra la enfermedad de Gumboro (Bursine 2, Fort Dodge) y contra la enfermedad de Newcastle y bronquitis infecciosa (Nobilis clone vac $30+$ MAS) vía ocular.

A los días 21 y 42 de edad, se evaluaron como variables de respuesta el peso corporal (g), consumo de alimento (g por ave por día), conversión alimenticia $\left(\mathrm{gg}^{-1}\right)$ y mortalidad acumulada (\%). Al final del experimento, se seleccionaron de forma aleatoria cuatros pollos por tratamiento (16 aves), y se identificaron en el tarso derecho y se sometieron a un ayuno de alimento de ocho horas; posteriormente, se pesaron y fueron sacrificados por dislocación cervical, con base en la NOM-033-ZOO-1995, para determinar el rendimiento de la canal. Se determinó el peso de la canal $(\mathrm{g})$, pechuga $(\mathrm{g})$ y pierna y muslo $(\mathrm{g})$.

Los datos fueron analizados por un diseño completamente al azar, para cada día de muestreo, por la ecuación: $Y_{\mathrm{ij}}=\mu+\mathrm{t}_{\mathrm{i}}+\mathrm{E}_{\mathrm{ij}}$, en que $\mathrm{Y}_{\mathrm{ij}}$ es la j-ésima observación asociada al i-ésimo tratamiento $(\mathrm{i}=1$, $2, \ldots \mathrm{t}),(\mathrm{j}=1,2, . . \mathrm{r}) ; \mu$ es la media poblacional; $t_{\mathrm{i}}$ es el efecto del j-ésimo tratamiento; $y \mathrm{E}_{\mathrm{ij}}$ es el error aleatorio $\operatorname{NID}\left(0, \sigma^{2}\right)$. 
Cuadro 1. Dietas experimentales de acuerdo a la fase de alimentación del pollo de engorda.

\begin{tabular}{|c|c|c|c|c|}
\hline \multirow[t]{2}{*}{ Ingredientes } & \multicolumn{2}{|c|}{ Iniciador 1 a 21 días } & \multicolumn{2}{|c|}{ Finalizador 22 a 42 días } \\
\hline & Testigo & Experimental & Testigo & Experimental \\
\hline Sorgo & 53,70 & 54,00 & 53,70 & 61,50 \\
\hline Pasta de soya & 28,50 & 29,00 & 28,50 & 23,00 \\
\hline Canola & 5,00 & 5,00 & 5,00 & 6,07 \\
\hline Ácidos Grasos & 4,34 & 4,25 & 4,34 & 3,00 \\
\hline Harina de carne & 3,00 & 3,00 & 3,00 & 2,00 \\
\hline Salvado de trigo & 2,10 & 1,80 & 2,10 & 1,70 \\
\hline Combinación harina ${ }^{(1)}$ & - & 0,07 & - & 0,07 \\
\hline Ortofosfato $(21 / 18)$ & 1,30 & 1,30 & 1,30 & 1,32 \\
\hline Carbonato de calcio $(38 \%)$ & 0,94 & 0,91 & 0,94 & 0,70 \\
\hline Cloruro de colina & 0,05 & 0,05 & 0,05 & 0,05 \\
\hline Sal refinada & 0,30 & 0,30 & 0,30 & 0,30 \\
\hline Premezcla de minerales ${ }^{(2)}$ & 0,05 & 0,05 & 0,05 & 0,05 \\
\hline Premezcla de vitamina ${ }^{(3)}$ & 0,03 & 0,03 & 0,03 & 0,03 \\
\hline DL-Metionina (99\%) & 0,24 & 0,24 & 0,25 & 0,22 \\
\hline L-Lisina $(78 \%)$ & - & - & 0,03 & 0,03 \\
\hline Flavomicina $(4 \%)$ & 0,25 & - & 0,25 & - \\
\hline Mycosor B & 0,10 & - & 0,10 & - \\
\hline Avatec (coccidiostato) & 0,05 & - & 0,05 & - \\
\hline Funginat (fungicida) & 0,05 & - & 0,05 & - \\
\hline Oxidox beta & 0,01 & - & 0,01 & - \\
\hline Total & 100,00 & 100,00 & 100,00 & 100,00 \\
\hline
\end{tabular}

El análisis se realizó con el PROC GLM del SAS (SAS Institute, 2001). La diferencia entre las medias de los tratamientos se analizó por medio de la prueba de rango múltiple de Tukey (Mendenhall, 1994), tomándose como diferencia mínima significativa $5 \%$ de probabilidad. Los resultados, expresados en porcentajes, fueron transformados a la función arcoseno raíz cuadrada para su análisis.

\section{Resultados y Discusión}

A los 21 días de edad, los pollos alimentados con OHS tuvieron el menor peso corporal y alta conversión alimenticia de los tratamientos durante esta fase $(\mathrm{p}<0,05)$. El testigo registró la mayor mortalidad a los 21 días de edad. Al final de la prueba (42 días), el mayor peso corporal lo obtuvo el testigo (flavomicina $4 \%$ ), que no presentó diferencia $(\mathrm{p}>0,05)$ con la combinación AO y tuvo la menor conversión de alimento $(1,96)$. La mayor viabilidad $(94,45 \%)$ fue en la combinación HSA (Cuadro 2).
Algunos autores han reportado efecto beneficioso con el uso de las combinaciones de harinas de hojas de plantas aromáticas similar a los encontrados en APC, en razón del eficiente uso de los nutrientes de la dieta, que se traduce en una menor conversión de alimento (Devriese et al., 1993; Lee et al., 2003). Kamel (2001) menciona que el desarrollo de vellosidades intestinales y el estímulo de la actividad enzimática están directamente involucrados y afectan el consumo de alimento. Lo anterior puede explicar los resultados similares $(p>0,05)$ en el consumo de alimento, a los 42 días, entre las harinas OA y HSA.

Esta eficiencia, de igual forma, se observó en las combinaciones de harinas que contenían albahaca (OA y HSA), en ambas fases de crecimiento, y favoreció la viabilidad de los mismos. Los APC mejoran la tasa de crecimiento (Devriese et al., 1993) del pollo y, en el presente estudio, se lo pudo observar. Sin embargo, las combinaciones OA y HSA, al presentar la mayor viabilidad de manera directa, incrementaron los 
kilogramos de aves por metro cuadrad, indicador de importancia en la industria avícola.

García Ríos et al. (2007) reportaron mayor ganancia de peso en los pollos alimentados con una combinación de aceites esenciales de orégano, canela, y pimienta, ricos en cinamaldehido, carvacrol y capsicum. Guo et al. (2004) obtuvieron resultados equivalentes a los extractos de virginiamicina y hierbas chinas. El uso y efecto de los aceites esenciales, en las dietas para pollos de engorda, han sido documentados en los últimos años (Lee et al., 2003). Los resultados encontrados en el presente estudio con harinas son similares a los reportados con aceites esenciales, pues comparten una estructura química similar llamada isopreno, unidad química de los terpenoides, que derivan en tres fenoles, con propiedades antimicrobianas: timol, carvacrol y eugenol. De acuerdo con la concentración de inclusión de los dos últimos llegan a disociarse las membranas externas de bacterias Gram negativas como Escherichea coli y Salmonella typhimurium. Las causas de mortalidad no fueron documentadas.
Los resultados del rendimiento de la canal se observan en el Cuadro 3. No se encontró diferencias $(p>0,05)$ entre el testigo y las combinaciones con OA y HSA en el peso de pechuga, muslo y pierna; el testigo registró menor porcentaje de rendimiento de la canal ( $>00,05$ ), en relación a las combinaciones de harina de hojas de las plantas aromáticas. Los datos encontrados en el peso de la pechuga, muslo y pierna coinciden con los reportados por Fukayama et al. (2005) sobre dietas adicionadas con extracto de orégano y APC. García et al. (2007) no reportan diferencias en dietas adicionadas con APC que tuvieron el menor rendimiento de la canal ( $p>0,05)$, con respecto a dietas combinadas con aceites esenciales de orégano, canela y pimienta. Los resultados obtenidos en el rendimiento de la canal se debió a un mayor aprovechamiento de los nutrientes de la dieta adicionada con la combinación de harina de hoja; el eugenol y cinamaldehido presentes estimulan la secreción de enzimas digestivas y favorecen la digestión y absorción intestinal que se mide como conversión

Cuadro 2. Comportamiento productivo de pollos de engorda, durante el ciclo de producción de 42 días, alimentados con harinas de plantas aromáticas ${ }^{(1)}$.

\begin{tabular}{lcccc}
\hline Tratamiento & $\begin{array}{c}\text { Peso corporal } \\
(\mathrm{g})\end{array}$ & $\begin{array}{c}\text { Consumo } \\
(\mathrm{g})\end{array}$ & $\begin{array}{c}\text { Conversión } \\
\left(\mathrm{g} \mathrm{g}^{-1}\right)\end{array}$ & $\begin{array}{c}\text { Mortalidad acumulada } \\
(\%)\end{array}$ \\
\hline
\end{tabular}

\begin{tabular}{|c|c|c|c|c|}
\hline \multirow[b]{2}{*}{ Testigo } & \multicolumn{4}{|c|}{21 días de edad } \\
\hline & $660,25 \pm 29,81 b$ & $121,86 \pm 11,31$ & $2,29 \pm 0,19 b$ & $17,18 \pm 3,12 \mathrm{a}$ \\
\hline OHS & $395,75 \pm 64,88 \mathrm{c}$ & $110,71 \pm 18,34$ & $3,53 \pm 0,66 a$ & $12,50 \pm 12,32 \mathrm{ab}$ \\
\hline $\mathrm{OA}$ & $785,50 \pm 35,12 \mathrm{a}$ & $109,90 \pm 7,34$ & $1,63 \pm 0,13 b$ & $2,78 \pm 3,21 b$ \\
\hline \multirow[t]{2}{*}{ HSA } & $745,50 \pm 23,02 \mathrm{ab}$ & $105,10 \pm 4,07$ & $1,70 \pm 0,06 b$ & $2,78 \pm 3,21 b$ \\
\hline & \multicolumn{4}{|c|}{42 días de edad } \\
\hline Testigo & $2.385 \pm 69,88 \mathrm{a}$ & $204,97 \pm 11,43 a$ & $2,17 \pm 0,02 b$ & $21,87 \pm 3,60 \mathrm{a}$ \\
\hline OHS & $1.750 \pm 163,70 \mathrm{c}$ & $158,67 \pm 17,44 b$ & $2,44 \pm 0,18 \mathrm{a}$ & $13,89 \pm 11,56 a b$ \\
\hline $\mathrm{OA}$ & $2.198 \pm 62,83 \mathrm{ab}$ & $160,74 \pm 13,04 b$ & $1,96 \pm 0,11 b$ & $8,33 \pm 5,55 b$ \\
\hline$\underline{\mathrm{HSA}}$ & $2.023 \pm 40,12 \mathrm{~b}$ & $174,33 \pm 7,10 \mathrm{~b}$ & $2,07 \pm 0,05 \mathrm{~b}$ & $5,55 \pm 6,41 b$ \\
\hline
\end{tabular}

Cuadro 3. Características de la canal de pollos de engorda, alimentados con harinas de plantas aromáticas ${ }^{(1)}$.

\begin{tabular}{lcccc}
\hline Tratamiento & $\begin{array}{c}\text { Canal } \\
(\mathrm{kg})\end{array}$ & $\begin{array}{c}\text { Pechuga } \\
(\mathrm{g})\end{array}$ & $\begin{array}{c}\text { Pierna y muslo } \\
(\mathrm{g})\end{array}$ & $\begin{array}{c}\text { Rendimiento de la canal } \\
(\%)\end{array}$ \\
\hline Testigo & $1,97 \pm 0,11 \mathrm{a}$ & $440,00 \pm 47,69 \mathrm{a}$ & $505,50 \pm 52,87$ & $47,99 \pm 1,26$ \\
OHS & $1,56 \pm 0,09 \mathrm{c}$ & $336,50 \pm 33,83 \mathrm{~b}$ & $435,00 \pm 39,68$ & $49,49 \pm 3,86$ \\
OA & $1,87 \pm 0,14 \mathrm{ab}$ & $435,00 \pm 24,57 \mathrm{a}$ & $478,00 \pm 37,70$ & $48,82 \pm 2,33$ \\
HSA & $1,67 \pm 0,06 \mathrm{bc}$ & $434,00 \pm 8,16 \mathrm{a}$ & $425,50 \pm 43,09$ & $51,46 \pm 2,90$ \\
\hline
\end{tabular}

(1)Medias con letras diferentes difieren entre sí por el test de Tukey al 5\% de probabilidad. Testigo flavomicina al 4\%; OHS, combinación harina de $35 \mathrm{~g}$ orégano y $35 \mathrm{~g}$ hierba santa; OA, combinación harina de $35 \mathrm{~g}$ orégano y $35 \mathrm{~g}$ albahaca; HSA, combinación harina de $35 \mathrm{~g}$ hierba santa y $35 \mathrm{~g}$ albahaca. 
alimenticia (Devriese et al., 1993; Hernández et al., 2006; Zekaria, 2006).

\section{Conclusiones}

1. Las combinaciones de harinas de hojas de orégano y albahaca (OA), e hierba santa y albahaca (HSA), adicionadas al $0,07 \%$ en la dieta de pollos de engorda, tienen un efecto similar a la flavomicina al $4 \%$, utilizada como promotor del crecimiento en la industria avícola.

2. Las combinaciones de harinas de plantas aromáticas disminuyen la conversión de alimento e incrementan viabilidad de la parvada.

\section{Referencias}

AYALA, L.; MARTÍNEZ, M.; ACOSTA, A.; DIEPPA, O.; HERNÁNDEZ, L. Una nota acerca del efecto del orégano como aditivo en el comportamiento productivo de pollos de ceba. Revista Cubana de Ciencia Agrícola, v.40, p.455-458, 2006.

CASTRO, M. Uso de aditivos en la alimentación de los animales monogástricos. Revista Cubana de Ciencia Agrícola, v.39, p.451$457,2005$.

DEVRIESE, L.A.; DAUBE, G.; HOMMEZ, J.; HAESEBROUCK, F. In vitro susceptibility of Clostridium perfringens isolated from farm animals to growth-enhancing antibiotics. Journal of Applied Bacteriology, v.75, p.55-57, 1993.

FUKAYAMA, E.H.; BERTECHINI, A.G.; GERALDO, A.; KATO, R.K.; MURGAS, L.D.S. Extrato de orégano como aditivo em rações para frangos de corte. Revista Brasileira Zootecnia, v.34, p.2316-2326, 2005.

GARCÍA RÍOS, A.; LEYVA, M.A.; MARTÍNEZ M., J.R.; STASHENKO, E.E. Determinación de la composición química y actividad antioxidante in vitro del aceite esencial de Piper auritum (Piperaceae) difundida en la costa Colombiana. Scientia et Technica, v.13, p.439-442, 2007.

GARCÍA, E. Modificación al sistema de clasificación climática de Köppen: para adaptarlo a las condiciones de la República Mexicana. México: UNAM, 1973. 246p.

GARCÍA, V.; CATALÁ-GREGORI, P.; HERNÁNDEZ, F.; MEGÍAS, M.D.; MADRID, J. Effect of formic acid and plant extracts on growth, nutrient digestibility, intestine mucosa morphology, and meat yield of broilers. Journal of Applied Poultry Research, v.16, p.555-562, 2007.

GUO, F.C.; WILLIAMS, B.A.; KWAKKEL, R.P.; LI, H.S.; LI, X.P.; LUO, J.Y.; LI, W.K.; VERSTEGEN, M.W.A. Effects of mushroom and herb polysaccharides, as alternatives for an antibiotic, on the cecal microbial ecosystem in broiler chickens. Poultry Science, v.83, p.175-182, 2004.

HERNÁNDEZ, F.; GARCÍA, V.; MADRID, J.; ORENGO, J.; CATALÁ, P. Effect of formic acid on performance, digestibility, intestinal histomorphology and plasma metabolite levels of broiler chickens. British Poultry Science, v.47, p.50-56, 2006.

HERNÁNDEZ, F.; MADRID, J.; GARCÍA, V.; ORENGO, J.; MEGÍAS, D. Influence of two plant extracts on broilers performance, digestibility, and digestive organ size. Poultry Science, v.83, p.169-174, 2004.

KAMEL, C. Tracing modes of action and the roles of plant extracts in non-ruminants. In: GARNSWORTHY, P.C.; WISEMAN, J. (Ed.). Recent advances in animal nutrition. Nottingham: Nottingham University Press, 2001. p.135-150.

KISER, J.S. A perspective on the use of antibiotics in animal feeds. Journal of Animal Science, v.42, p.1058-1072, 1976.

LEE, K.-W.; EVERTS, H.; KAPPERT, H.J.; YEOM, K.-H.; BEYNEN, A.C. Dietary carvacrol lowers body weight gain but improves feed conversion in female broiler chickens. Journal of Applied Poultry Research, v.12, p.394-399, 2003.

MENDENHALL, W. Introduction to probability and statistics. In: MENDENHALL, W.; BEAVER, R.J. (Ed.). Introduction to linear models and the design and analysis of experiments. Belmont: Wadsworth Publlishing Company, 1994. p.244-251.

NATIONAL RESEARCH COUNCIL. Nutrient requirements of poultry. $10^{\text {th }}$ ed. Washington: National Academy of Sciences, 1994. $155 \mathrm{p}$

PLAUS, E.A.; FLORES, G.S.; ATAUCUSI, S.G. Composición química y actividad antibacteriana del aceite esencial del Origanum vulgare (orégano). Revista Medica Herediana, v.12, p.16-19, 2001.

SAS INSTITUTE. Statistical analysis system: user's guide. Version 8.2. Cary: SAS Institute, 2001.

ZEKARIA, D. Los aceites esenciales: una alternativa a los antimicrobianos. 2006. Disponible en: < http://www.calier.es/pdf/ Microsoft_Word_-_Aceites_esen_como_promotores.pdf $>$. Leído el: 05 mayo 2010 .

Recibido el 29 de diciembre de 2009 e aprobado el 5 de febrero de 2010 